\title{
Integration of reliability-based topology optimization into biomechanics: Application on hollow stems used in cementless total hip arthroplasty
}

\author{
Ghais Kharmanda ${ }^{1}$, Imad Antypas ${ }^{2, *}$, and Alexey Dyachenko ${ }^{2}$ \\ ${ }^{1}$ INSA Rouen, , 76800, St Etienne du Rouvray, France \\ ${ }^{2}$ Don State Technical University, 344003, Gagarin sq., 1, Rostov on Don, Russian Federation
}

\begin{abstract}
The integration of topology optimization into biomechanical applications possesses an important key to increase the performance levels. In literature, two models can be found: Deterministic Topology Optimization (DTO) and Reliability-Based Topology Optimization (RBTO). The DTO leads to a single layout for a given design space. However, the RBTO generates several reliability-based topologies with high performance levels. Topology optimization has been already integrated into biomechanical applications such as prosthesis design. The filter-based approaches being tied to the element discretization, are previously used to control the resulting topologies or to prevent undesirable designs. These are expensive operations for fine meshes or complex domains and numerous numerical difficulties can be met. In this work, the RBTO is integrated at the conceptual design stage of the total hip replacement to control the resulting topologies to meet different constraints such as manufacturing constraints. This can provide several types of hollow stems considering the daily loading cases. Two alternative approaches based on the Optimum Safety Factors (OSF) are developed to provide two categories of solutions. The RBTO model can be integrated into the additive manufacturing technology as a topology generator satisfying several manufacturing constraints. The resulting different configurations can then be provided for various patients.
\end{abstract}

\section{Introduction}

In literature, topology optimization can be classified into two main models: deterministic model and reliability-based one. In Deterministic Topology Optimization (DTO), a single solution for a given domain can be reached. However, the Reliability-Based Topology Optimization (RBTO) model developed in this paper can generate several solutions with different advantages. It allows us to select the best solution to fulfill the technical specifications. The structural weight of the resulting topologies obtained by this model is reduced when comparing with the DTO model. Furthermore, when the RBTO model leads

\footnotetext{
*Corresponding author: imad.antypas@mail.ru
} 
to more reliable structures compared to the DTO model for the same weight levels [1]. Two points of view are presented for the RBTO developments: topology optimization [2-4] and reliability analysis $[5,6]$. Considering previous works, it has been shown that the point of view 'topology optimization' seems to be interesting for structural designers since it provides several reliability-based structures relative the reliability index changes. It leads to different layout structures while the developments from a point of view 'reliability analysis' leads to same configurations with different densities that have no sense for integrating the reliability concept.

To perform the RBTO problems, several RBDO approaches can be adopted since we deal with different philosophies. The RBDO methods have been already developed regarding to their use [1]. For example, the OSF method has been simply implemented by performing two main procedures. The first one is to find the failure point performing a simple optimization loop while the second one consists of using the OSF formulations to compute the optimum solution. In this work, the OSF method is adopted in another way for the RBTO model, the developed Inverse Optimum Safety (IOSF) method consists of first reaching the configuration using Deterministic Topology Optimization (DTO). Next the OSF formulations are used with inverse derivative signs in order to generate several reliability-based topologies. The integration of the DTO and RBTO into hip prosthesis stems is motivated as a practical case. These models are considered as effective tools for the conceptual design stage to delete un-needed structural region and also to generate transversal holes allowing a good fixation level after the healing period.

\section{Materials and Methods}

\subsection{Deterministic Topology Optimization}

The problem of topology optimization is described here in two different ways. The first way is to minimize the structural compliance respecting a target ratio decrease of volume $V_{f}^{\text {Tar }}$. The problem of DTO model is then mathematically described by:

$$
\begin{gathered}
\min : \operatorname{Com}(x) \\
\text { s.t.: } \frac{\operatorname{Vol}(x)}{V^{0}} \leq V_{f}^{\mathrm{Tar}}
\end{gathered}
$$

where $\operatorname{Com}(x)$ is the structural compliance considering the densities of the used material in each element as optimization variables. Their values should be found in the interval $[0,1]$. $V^{0}$ and $\operatorname{Vol}(x)$ are the initial- and current structural volume values. Conversely, the second one is to minimize the structural volume under a target ratio increase of compliance $C_{f}^{\mathrm{Tar}}$. The DTO problem is then mathematically written as follows:

$$
\begin{gathered}
\min : \operatorname{Vol}(x) \\
\text { s.t.: } \frac{\operatorname{Com}(x)}{C^{0}} \leq 1+C_{f}^{\mathrm{Tar}}
\end{gathered}
$$

where $\operatorname{Vol}(x)$ is the structural volume considering the material densities in each element as optimization variables and belong to the interval $[0,1] . C^{0}$ and $\operatorname{Com}(x)$ represent the initialand current structural compliance values. Formulations 1 and 2 are basic forms and can be used considering several topology optimization strategies such as SIMP (Solid Isotropic Microstructure with Penalty), homogenization approach [7]. 


\subsection{Reliability Index}

In order to produce different topologies, a reliability index $\beta$ is used with a normalized vector $\mathbf{u}$. Fig. 1 shows the transformation image between the random variables $\mathbf{y}$ in the physical space and the normalized vector $\mathbf{u}$ in the normalized space [8]. The reliability index assessment is carried out using FORM (First Order Reliability Method). The evaluation of the reliability index can be performed using an optimization procedure:

$$
\beta=\min d(u) \text { s.t.: } H(u)=0
$$

where the distance $d(u)$ in the normalized space is given by:

$$
d(u)=\sqrt{\sum_{i=1}^{n} u_{i}^{2}}
$$

This procedure seeks to find the optimum value of $\beta$ which corresponds to the Most Probable failure Point (MPP). This point is represented by the minimum distance between the origin of the normalized space and limit state curve as shown in Fig. 1. In structural engineering, the reliability index interval has to be: $\beta \in[3-4.25]$.

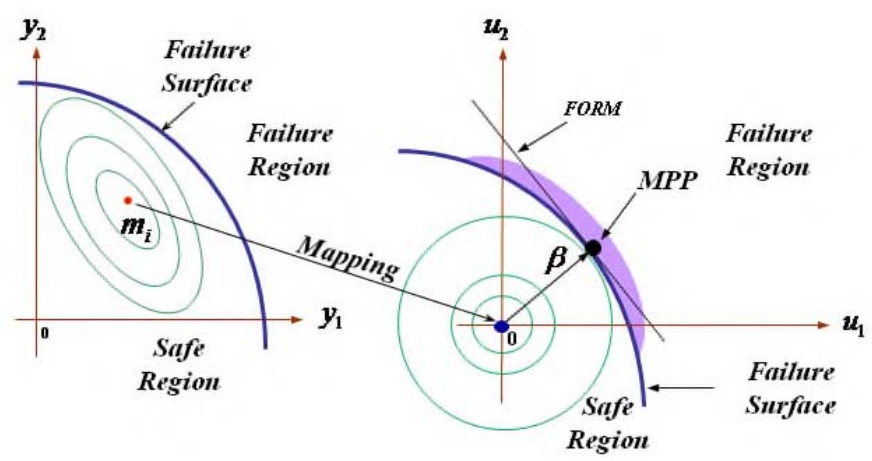

Fig. 1. Transformation image between the physical space and the normalized one

\subsection{Reliability-Based Topology Optimization}

The previous OSF strategy can efficiently decrease the size of the RBDO problem using the sensitivities of the limit state function with respect to all the structure's parameters, especially when the sensitivity can be calculated analytically [9]. The main idea of OSF strategy is to find the failure point and next the optimum solution using the OSF formulations. In the RBTO, it is the objective to evaluate the starting point and next to find the reliability-based points. Two approaches are developed here.

\subsubsection{Objective-Based IOSF Approach}

The problem of RBTO model is to minimize the structural compliance under a target ratio decrease of volume $V_{f}^{\mathrm{Tar}}$ and the reliability constraint. The RBTO problem is then mathematically written as: 


$$
\begin{gathered}
\min : \operatorname{Com}(x) \\
\text { s.t.: } \beta \geq \beta_{t} \\
\text { and: } \frac{\operatorname{Vol}(x)}{V^{0}} \leq V_{f}^{\mathrm{Tar}}
\end{gathered}
$$

where $\beta_{t}$ is the required reliability index to be satisfied. The failure is generally related to the compliance. However, in this method, the structural compliance is considered as an objective function. Thus, the sensitivity estimation is carried out for the objective function which is considered as a failure criterion. So, the optimum value of the normalized vector can be written by:

$$
u_{i}= \pm \beta_{t} \sqrt{\frac{\left|\frac{\partial F}{\partial y_{i}}\right|}{\sum_{j=1}^{n}\left|\frac{\partial F}{\partial y_{j}}\right|}}
$$

Considering the derivative sign of the objective function with respect to random variables $y_{i}$, we write:

$$
\frac{\partial F}{\partial y_{i}}>0 \Leftrightarrow u_{i}>0 \text { and } \frac{\partial F}{\partial y_{i}}<0 \Leftrightarrow u_{i}<0
$$

In this approach, the OSF concepts and the failure criterion are taken into account. When considering the failure criterion as an objective function, the Objective-Based IOSF Approach can be performed, but when the failure criterion is treated as a performance function, the method can be called Performance-Based IOSF Approach being presented in the following section.

\subsubsection{Performance-Based IOSF Approach}

The RBTO problem consists of minimizing the structural volume under the compliance constraint and the reliability one. The RBTO problem is then mathematically written as:

$$
\begin{gathered}
\min : \operatorname{Vol}(x) \\
\text { s.t.: } \beta \geq \beta_{t} \\
\text { and: } \frac{\operatorname{Com}(x)}{C^{0}} \leq 1+C_{f}^{\text {Tar }}
\end{gathered}
$$

The optimum value of the normalized vector can be expressed by:

$$
u_{i}= \pm \beta_{t} \sqrt{\frac{\left|\frac{\partial G}{\partial y_{i}}\right|}{\sum_{j=1}^{n}\left|\frac{\partial G}{\partial y_{j}}\right|}}
$$

According to the derivative sign of the limit state function with respect to random variables $y_{i}$, we write:

$$
\frac{\partial G}{\partial y_{i}}>0 \Leftrightarrow u_{i}>0 \text { and } \frac{\partial G}{\partial y_{i}}<0 \Leftrightarrow u_{i}<0
$$




\subsection{Probabilistic distribution laws}

Several distribution laws can be used, for simplicity, the random variable vector is considered here to be normally distributed, the safety factor can be expressed by:

$$
S_{f_{i}}=1+\gamma_{i} u_{i}
$$

where $\gamma_{i}$ represents the relationship between the mean $m_{i}$ and standard-deviation $\sigma_{i}$ as follows:

$$
\gamma_{i}=\sigma_{i} / m_{i}
$$

In both developed approaches, it is considered that the starting point represents the failure point $P_{y}$ and next the reliability-based topology $P_{x}$ is found. The reliability-based topology $P_{x}$ must be more reliable than the failure point $P_{y}$ and must also verify a required reliability index $\beta_{t}$. This way the DTO procedure is used to find the failure point $P_{y}$ and the both developed approaches are then used to determine the reliability-based topologies $P_{x}$.

\subsection{Integration of RBTO into biomechanics}

\subsubsection{Importance}

The integration of reliability-based topology optimization into biomechanics can be carried out on several biomechanical applications such as orthopedic and orthodontic prostheses design [1]. In general, the selection of an appropriate structural topology possesses a very important effect on the structure's future performance levels. As a result, topology optimization possesses a significantly key place in the preliminary decision-making procedure at the design stage. Topology optimization can be considered as a design tool to select an appropriate initial topology. In addition to the topology optimization importance, the RBTO provides us with several topologies that can meet various patient specifications.

\subsubsection{Integration of RBTO into total hip replacement}

The RBTO can be integrated at the conceptual design stage to sketch several types of hollow stems according to the daily loading cases [10-12]. In this section, a 2D model is considered for simplicity to carry out the different topology optimization studies [12]. As shown in Fig. 2a, the studied stem is surrounded by two bone tissues (cortical and cancellous tissues). The material properties, boundary conditions and optimization domain are presented here.

\subsubsection{Material properties}

The cortical bone tissue is assumed to be a homogeneous and isotropic material with Young's modulus $E=17 \mathrm{GPa}$ and Poisson's ratio $v=0.33$. Moreover, the cancellous bone tissue is assumed to be a homogeneous and isotropic material with Young's modulus $E=386 \mathrm{MPa}$ and Poisson's ratio $v=0.33$ [13]. The modulus of titanium alloy of stem is considered to be: $E=110 \mathrm{GPa}$ with Poisson's ratio: $v=0.3$ [14]. 

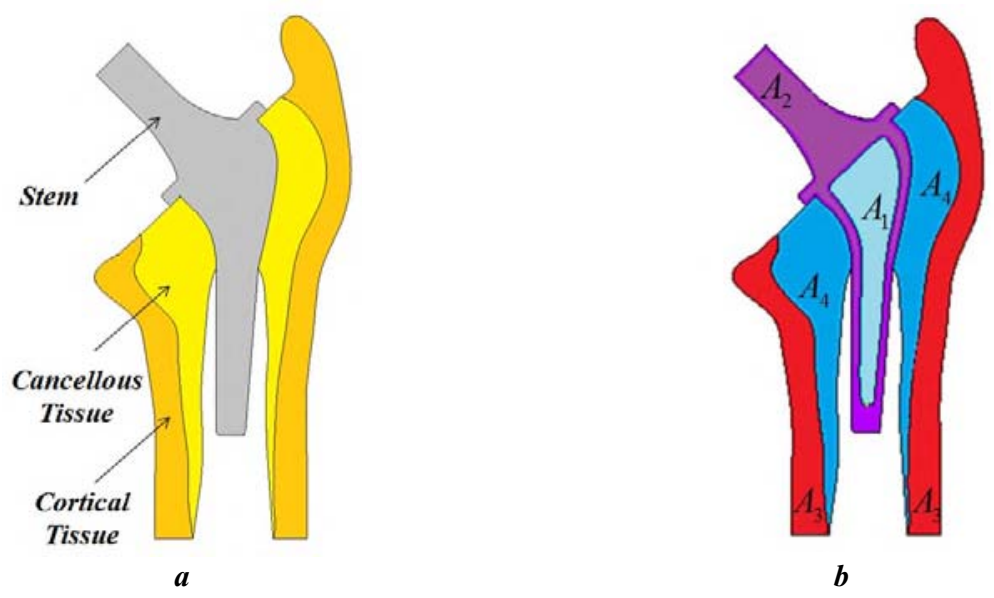

Fig. 2. a) 2D studied stem model implanted in bone tissues and b) Inner area as an optimized domain

\subsubsection{Optimized domain}

The topology optimization algorithm allows us to delete un-needed regions especially at the border which is in contact with the bone tissue. Thus, the outer border should not considered as optimization domain. It is the objective to specify optimized and nonoptimized regions for the shaft in the stem component. The inner area of the shaft $A_{1}$ is assumed to be the optimized domain, while the other areas to be non-optimized domains: $A_{2}$ contains the head, neck, shoulder and an outer area of the shaft, $A_{3}$ contains the cortical tissue and $A_{4}$ contains the cancellous tissue.

\subsubsection{Boundary conditions}

Three representative daily loading cases are assumed: one-legged stance $(L 1)$, extreme ranges of motion of abduction (L2), and adduction (L3) [15], as shown in Fig. 3.

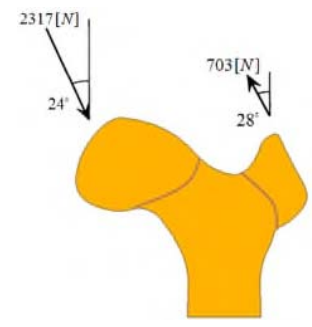

$a$

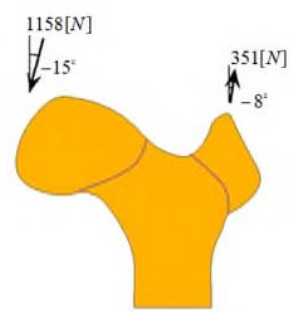

$b$

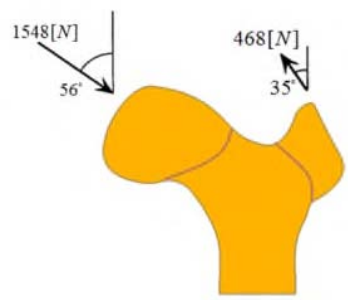

$\boldsymbol{c}$

Fig. 3. a) One-legged stance case (L1), b) Abduction case (L2), and c) Adduction case (L3)

\section{Results and discussion}

Figs. 4, 5 and 6 correspond to different resulting topologies for different loading cases: $L 1$, $L 2$ and $L 3$, respectively. The colors present the materials densities where the red color means an element with full material which can be represented by a solid element. The 
colors change according to the density to become blue which can be represented by a void element. DTO and RBTO models are considered for comparison. The reliability index values belong to the interval $\beta \in[3-4.25]$. Three values are selected to identify the effect of the layout changes: $\beta=3,3.8$ and 4.25. All numerical DTO and RBTO results are presented in Table 1 considering the developed approaches. The structural compliance and volume values change in function with the reliability index values for the three considered loading cases, however the presented figures correspond to only the layouts changes.

Table 1. Numerical DTO and RBTO results

\begin{tabular}{|c|c|c|c|c|c|c|c|c|c|}
\hline \multirow{3}{*}{\multicolumn{2}{|c|}{$\begin{array}{c}\text { Case } \\
\& \\
\text { Model }\end{array}$}} & \multicolumn{4}{|c|}{ First Category } & \multicolumn{4}{|c|}{ Second Category } \\
\hline & & \multirow{2}{*}{ DTO } & \multicolumn{3}{|c|}{ RBTO } & \multirow{2}{*}{ DTO } & \multicolumn{3}{|c|}{ RBTO } \\
\hline & & & $\beta=3$ & $\beta=3.8$ & $\beta=4.25$ & & $\beta=3$ & $\beta=3.8$ & $\beta=4.25$ \\
\hline \multirow{2}{*}{$L 1$} & Com & 0.74 & 0.95 & 1.01 & 1.05 & 1.07 & 1.97 & 2.28 & 2.45 \\
\hline & Vol & 351.37 & 321.77 & 313.88 & 309.43 & 149.66 & 89.96 & 85.03 & 85.85 \\
\hline \multirow{2}{*}{$L 2$} & Com & 0.72 & 0.85 & 0.91 & 0.93 & 0.95 & 1.04 & 1.07 & 1.08 \\
\hline & Vol & 351.38 & 383.62 & 392.22 & 397.06 & 107.22 & 132.39 & 134.24 & 134.12 \\
\hline \multirow{2}{*}{$L 3$} & Com & 0.37 & 0.77 & 0.91 & 1.01 & 0.48 & 0.94 & 1.10 & 1.20 \\
\hline & Vol & 351.38 & 307.89 & 296.30 & 289.78 & 264.70 & 253.94 & 249.72 & 246.30 \\
\hline
\end{tabular}

As shown in Fig. 4 for L1, the first approach (Objective-Based IOSF Approach) does not lead to a significant layout change even when arriving to the end of the interval $(\beta=4.25)$. When increasing the reliability index values, the structural compliance increases, while the structural volume decreases as shown in Table 1. On the other hand, the second approach (Performance-Based IOSF Approach) leads a change even at the beginning of the interval $\beta=3$. When increasing the reliability index values, the structural compliance increases, while the structural volume decreases except for $\beta=4.25$ as shown in Table 1.
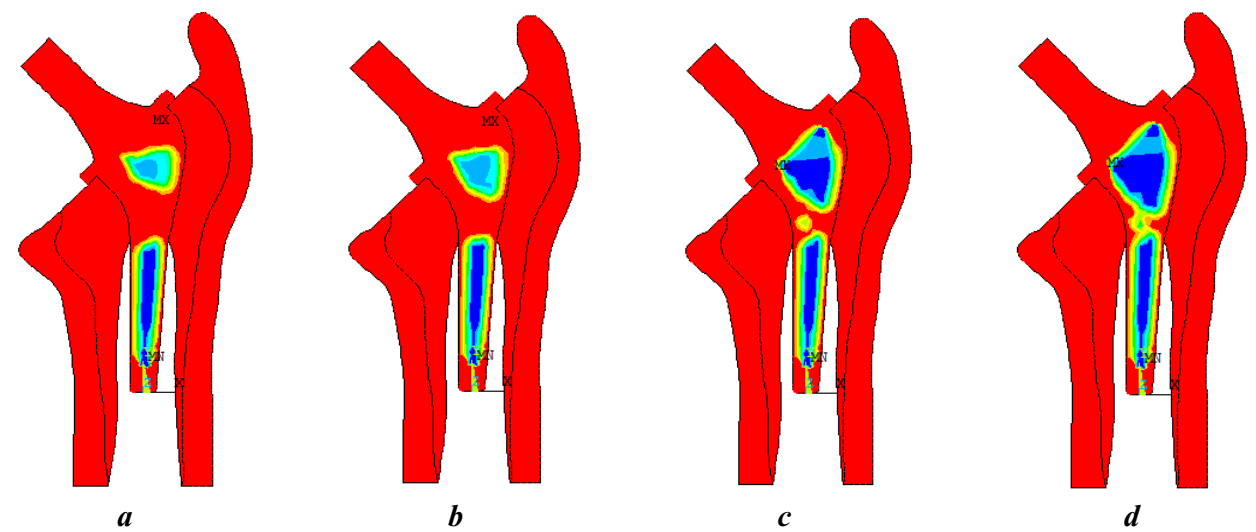

Fig. 4. a) DTO and b) RBTO layouts when using the first approach, c) DTO and d) RBTO layouts when using the second approach, for the first loading case $L 1$.

As shown in Fig. 5 for $L 2$, the first approach leads to a significant change when the reliability index is: $\beta=3.8$. When increasing the reliability index values, the structural compliance and volume increase as shown in Table 1. On the other hand, the second approach does not lead to a significant change even when arriving to the end of the interval $(\beta=4.25)$. When increasing the reliability index values, the structural compliance and volume increase except for $\beta=4.25$ where the structural volume decreases as shown in Table 1. 


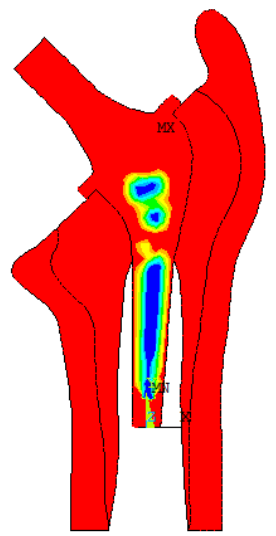

$\boldsymbol{a}$

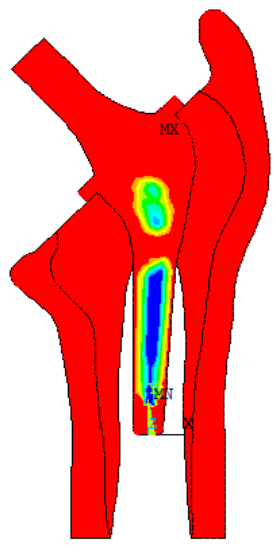

$\boldsymbol{b}$

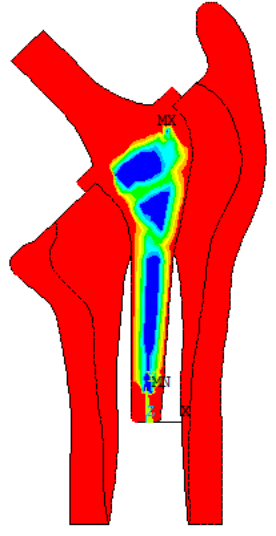

$c$

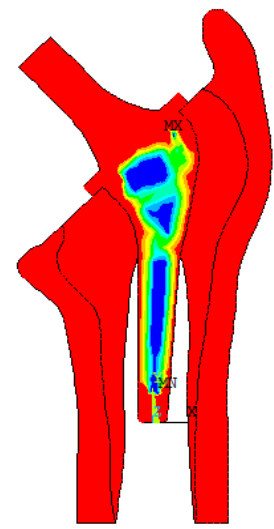

$d$

Fig. 5. a) DTO and b) RBTO layouts when using the first approach, c) DTO and d) RBTO layouts when using the second approach, for the second loading case $L 2$.

As shown in Fig. 6 for $L 3$, the first approach does not lead to a significant change even when arriving to the end of the interval $(\beta=4.25)$. When increasing the reliability index values, the structural compliance increases, while the structural volume decreases as shown in Table 1. On the other hand, the second approach leads a small change at the end of the interval $(\beta=4.25)$. When increasing the reliability index values, the structural compliance increases, while the structural volume decreases as shown in Table 1.

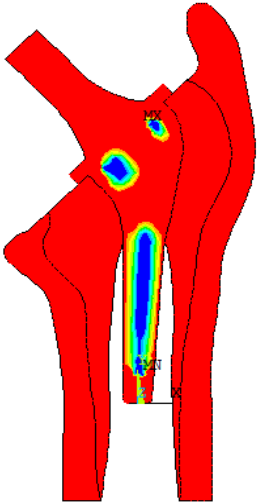

$a$

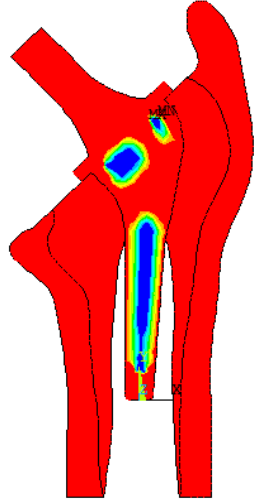

$b$

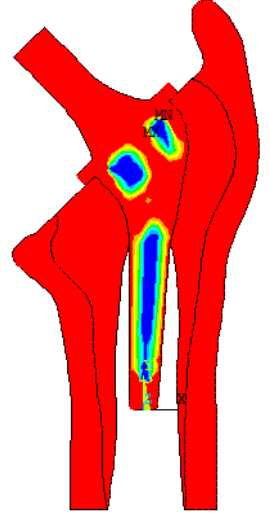

$c$

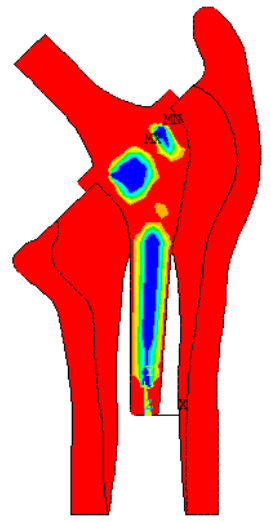

$d$

Fig. 6. a) DTO and b) RBTO layouts when using the first approach, c) DTO and d) RBTO layouts when using the second approach, for the third loading case $L 3$.

According to Table 1, for both approaches and for all loading cases, when increasing the reliability index values, the structural compliance values increase, while the structural volume values decrease except for the second loading case L2. It is very important to consider both approaches during the prosthesis design stage since the first approach provides us with different layouts in certain loading cases (see Figs 5a and b), while the second approach do that with the other loading cases (see Figs $4 \mathrm{c}$ and d). 


\section{Conclusions}

The integration of the RBTO is carried out into the total hip replacement in order to provide several kinds of hollow stems with object of increasing the prosthesis performance, especially osseointegration. The uncertainty is applied on the material properties, loads and constraint boundaries. Two RBTO approaches are developed here and lead in certain cases to different layouts compared to the DTO configurations. It is very important to consider the uncertainty on the stem geometry in order to generate new layouts with significant difference. The RBTO can be integrated to perform the additive manufacturing of the orthopedic prosthesis to meet the specifications of various patients. It may pave the way to many biomechanical applications.

\section{References}

1. G. Kharmanda, El-Hami, A., Biomechanics: Optimization, Uncertainties and Reliability, ISTE \& Wiley, ISBN: 9781786300256, 254 (2017)

2. J. Patel, S. K. Choi, Structural and Multidisciplinary Optimization, 45(4), 529-543 (2012) DOI: 10.1007/s00158-011-0711-2

3. L. Wang, D. Liu, Y. Yang, X. Wang, Z. Qiu, Computer Methods in Applied Mechanics and Engineering, 326, 573-595 (2017) DOI: 10.1016/j.cma.2017.08.023

4. G. Kharmanda, I. Antypas, A. Dyachenko, Journal of Engineering Technologies and Systems, 29(1), 8-19 (2019) DOI: 10.15507/2658-4123.029.201901.008-019

5. Y. S. Eom, K. S. Yoo, J. Y. Park, S. Y. Han, Structural and Multidisciplinary Optimization, 43(2), 287-295 (2011) doi: 10.1007/s00158-010-0569-8

6. M. alalpour, M. Tootkaboni, Journal of Structural and Multidisciplinary Optimization, 53, 759-772 (2016) DOI:10.1007/S00158-015-1360-7

7. W. Zhang, J. Zhu, T. Gao, Topology Optimization in Engineering Structure Design, ISTE \& Elsevier, ISBN: 9781785482243, 294 (2016)

8. G. harmanda, A. El-Hami, Reliability in Biomechanics, ISTE \& Wiley, ISBN: 9781786300249, 266 (2016)

9. O. Sigmund, K. Maute, Structural and Multidisciplinary Optimization, 48, 1031-1055 (2013) https://doi.org/10.1007/s00158-013-0978-6.

10. G. Kharmanda, I. Antypas, A. Dyachenko, International Journal of Mechanical Engineering Technology, 9(11), 810-820 (2018)

11. G. Kharmanda, I. Antypas, A. Dyachenko, International Journal of Mechanical Engineering Technology, 10(9), 1-11 (2019)

12. G. Kharmanda, I. Antypas, A. Dyachenko, E3S Web of Conferences, 175(14), (2020) DOI: $10.1051 / \mathrm{e} 3$ sconf/202017512004.

13. G. Kharmanda, Computer Methods in Biomechanics and Biomedical Engineering, 19(14), 1557-1566 (2016)

14. S. A. Shaik, K. Bose, H. P. Cherukuri, Materials and Design, 42, 230-237 (2012) DOI: 10.1016/j.matdes.2012.05.049

15. G. Kharmanda, Materials and Design, 65, 496-504 (2015) 